\title{
Large-Scale Simulation of Energetic Particle Driven Magnetohydrodynamic Instabilities in ITER Plasmas*)
}

\author{
Yasushi TODO ${ }^{1,2)}$ and Andreas BIERWAGE ${ }^{3)}$ \\ ${ }^{1)}$ National Institute for Fusion Science, Toki 509-5292, Japan \\ ${ }^{2)}$ The Graduate University for Advanced Studies (SOKENDAI), Toki 509-5292, Japan \\ 3) Japan Atomic Energy Agency, Rokkasho 039-3212, Japan \\ (Received 10 December 2013 / Accepted 13 March 2014)
}

\begin{abstract}
Magnetohydrodynamic (MHD) instabilities driven by energetic alpha particles and beam deuterium particles are investigated for ITER operation scenarios using a hybrid simulation code for energetic particles interacting with an MHD fluid. The particle simulation method with finite Larmor radius effects is applied to both alpha and beam deuterium particles. For the steady-state scenario with $9 \mathrm{MA}$ plasma current, beta-induced Alfvén eigenmodes (BAE modes) with low toroidal mode number $(n=3,5)$ were found to become dominant in the nonlinear phase although many toroidal Alfvén eigenmodes (TAE modes) with $n \sim 15$ are most unstable in the linear phase. The redistribution of energetic particles with $\delta \beta_{\alpha} \sim \delta \beta_{\text {beam }} \sim 0.07 \%$, which respectively correspond to $6 \%$ and $8 \%$ of the central values, occurs in the nonlinear phase. When the toroidal mode number of the fluctuations is restricted to $n \leq 8$, the redistribution is substantially reduced, thus, suggesting that the resonance overlap between the $n \sim 15$ TAE and low- $n$ BAE modes enhances the energetic particle transport in the run with full toroidal mode numbers. For the ITER scenario with 15 MA plasma current, an MHD instability with $n=3$ that peaks around the $q=1$ ( $q$ is the safety factor) magnetic surfaces is driven by bulk plasma current and bulk pressure, and results in significant redistribution of alpha particles with $\delta \beta_{\alpha} \sim 0.3 \%$. For the equilibrium profile with the safety factor profile uniformly raised by 0.1 to remove the $q=1$ surfaces, only a benign MHD instability occurs and the energetic particle transport is negligible.
\end{abstract}

(c) 2014 The Japan Society of Plasma Science and Nuclear Fusion Research

Keywords: ITER, Alfvén eigenmode, MHD instability, alpha particle, beam ion

DOI: $10.1585 /$ pfr.9.3403068

\section{Introduction}

Enhanced transport and losses of energetic particles owing to the Alfvén eigenmodes (AE modes) are of concern in burning plasma devices such as ITER. Beam ions with a maximum energy of $1 \mathrm{MeV}$ may destabilize the toroidal Alfvén eigenmodes (TAE modes) with the toroidal mode number $n \sim 10$ in an ITER plasma [1]. Nonlinear hybrid simulations of energetic particles interacting with magnetohydrodynamic (MHD) fluid showed that the AE modes with a low toroidal mode number $n=2-3$ are most unstable, and the redistribution of alpha particles is benign for ITER-like plasmas [2,3]. Recently, reversed shear Alfvén eigenmodes (RSAE modes) and TAE modes were found to be unstable with maximum growth rates for $n=6$ in ITER steady-state plasma [4]. In this study, we present the simulation results of ITER plasmas using MEGA [5-7], which is a hybrid simulation code for energetic particles interacting with an MHD fluid. The finite Larmor radius effect, which reduces the energetic particle drive to AE modes, has been implemented in the MEGA code. The MEGA code was benchmarked for the TAE

author'se-mail: todo@nifs.ac.jp

*) This article is based on the invited presentation at the 23rd International Toki Conference (ITC23). mode problem constructed by the Energetic Particle Topical Group of the International Tokamak Physics Activity. The growth rate of the TAE mode among the nine simulation codes, including MEGA, was found in good agreement [8]. For predicting the AE modes in ITER, investigating the realistic equilibrium using sufficient numerical resolution is important. We use the equilibrium data provided on the ITER IDM DATA folder. The equilibrium was constructed using the ASTRA [9] and EFIT [10] codes. The number of grid points is 30 times larger than that of our previous work [3] and is sufficient to resolve the spatial profile of AE modes with $n \geq 10$.

\section{Simulation Model}

Several hybrid simulation models have been constructed [11-15] to study the evolution of AE modes destabilized by energetic particles. In the MEGA code, the bulk plasma is described using nonlinear MHD equations, and the energetic ions are simulated with the $\delta f$ particle method. The MHD equations with the energetic ion effects are given by

$$
\frac{\partial \rho}{\partial t}=-\nabla \cdot(\rho \boldsymbol{v})+v_{\mathrm{n}} \Delta\left(\rho-\rho_{\mathrm{eq}}\right)
$$




$$
\begin{aligned}
\rho \frac{\partial}{\partial t} \boldsymbol{v}= & -\rho \vec{\omega} \times \boldsymbol{v}-\rho \nabla\left(\frac{v^{2}}{2}\right)-\nabla p+\left(\boldsymbol{j}-\boldsymbol{j}_{h}^{\prime}\right) \times \boldsymbol{B} \\
& +\frac{4}{3} \nabla(v \rho \nabla \cdot \boldsymbol{v})-\nabla \times(v \rho \vec{\omega}), \\
\frac{\partial \boldsymbol{B}}{\partial t}= & -\nabla \times \boldsymbol{E}, \\
\frac{\partial p}{\partial t}= & -\nabla \cdot(p \boldsymbol{v})-(\gamma-1) p \nabla \cdot \boldsymbol{v}+(\gamma-1)\left[v \rho \omega^{2}\right. \\
& \left.+\frac{4}{3} v \rho(\nabla \cdot \boldsymbol{v})^{2}+\eta \boldsymbol{j} \cdot\left(\boldsymbol{j}-\boldsymbol{j}_{\mathrm{eq}}\right)\right]+\chi \Delta\left(p-p_{\mathrm{eq}}\right), \\
& \boldsymbol{E}=-\boldsymbol{v} \times \boldsymbol{B}+\eta\left(\boldsymbol{j}-\boldsymbol{j}_{\mathrm{eq}}\right), \\
\boldsymbol{j}= & \frac{1}{\mu_{0}} \nabla \times \boldsymbol{B}, \\
\vec{\omega}= & \nabla \times \boldsymbol{v},
\end{aligned}
$$

where $\mu_{0}$ is the vacuum magnetic permeability, $\gamma$ is the adiabatic constant, $v, v_{\mathrm{n}}$, and $\chi$ are the artificial viscosity and diffusion coefficients for maintaining numerical stability, and all other quantities are conventional. In this study, the dissipation coefficients $v, v_{\mathrm{n}}, \chi$, and $\eta / \mu_{0}$ are equal to $10^{-7} v_{\mathrm{A}} R_{0}$ unless otherwise specified. The subscript "eq" denotes the equilibrium variables. The energetic ion contribution is included in the MHD momentum equation [Eq. (2)] as the energetic ion current density $\boldsymbol{j}_{h}^{\prime}$ that includes the contributions from parallel velocity, magnetic curvature and gradient drifts, and magnetization current. The $\boldsymbol{E} \times \boldsymbol{B}$ drift disappears in $\boldsymbol{j}_{h}^{\prime}$ owing to quasi-neutrality [4]. The electromagnetic field is given by the standard MHD description. This model is accurate under the condition that the energetic ion density is much less than the bulk plasma density. The MHD equations are solved using a fourth-order in the space and time finite-difference scheme.

The energetic alpha particles and beam deuterium ions are simulated using the $\delta f$ particle-in-cell method. We use the gyrokinetic approach to consider the finite Larmor radius effects. Electromagnetic fluctuations are averaged on the energetic particle gyro orbit for energetic particle dynamics. The equations of the guiding-center motion are given in Refs. $[6,7,16]$. The alpha particle distribution is isotropic in velocity space, whereas the beam deuterium particles are anisotropic. The anisotropy of the beam deuterium particles is considered by a distribution function model that is proportional to $\exp \left[-\left(\Lambda-\Lambda_{0}\right)^{2} / \Delta \Lambda^{2}\right]$ with $\Lambda=\mu B_{0} / E, \Lambda_{0}=0.3$, and $\Delta \Lambda=0.3 . E$ is the particle kinetic energy, and $B_{0}$ is the magnetic field strength at the magnetic axis. Cylindrical coordinates $(R, \phi, z)$ are used and $(256,256,512)$ grid points are allocated, respectively. The number of computational particles for each energetic alpha particle and beam deuterium ion is $N=8.4 \times 10^{6}$. The numerical convergence with particle number is discussed in subsection 3.2. For data analysis, magnetic flux coordinates $(r, \phi, \vartheta)$, where $\vartheta$ is the poloidal angle, were constructed for the investigated MHD equilibria. The guiding-center motion and the evolution of $\delta f$ for each computational particle are solved using a fourth-order

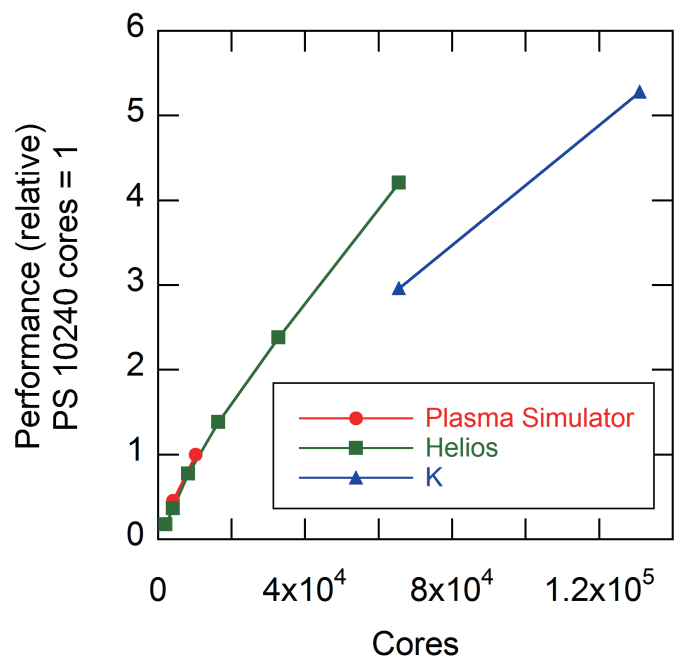

Fig. 1 Strong scaling of the MEGA code on Plasma Simulator (SR16000 M1), Helios, and K Computer. Good strong scaling is found beyond 105 cores on the $\mathrm{K}$ computer.

Runge-Kutta method.

The strong scaling of the MEGA code with the numbers of grid points $(512,512,320)$ and 670 million computational particles was investigated on Plasma Simulator, Helios, and K Computer. The results are shown in Fig. 1. On the K computer, we observed a good strong scaling beyond $10^{5}$ cores.

\section{Simulation Results}

\subsection{Steady-state scenario with 9 MA plasma current}

First, the steady-state scenario with 9 MA plasma current was investigated. We used equilibrium data obtained with the ASTRA and EFIT codes, and data stored on the ITER IDM DATA folder [17]. The equilibrium plasma profile is shown in Fig. 2. The magnetic shear profile is weakly reversed, and the $q$ minimum value is less than 2 . The central temperature for the ions and electrons is higher than $30 \mathrm{keV}$. The central beta values for the energetic alpha particles and beam deuterium ions are $1.3 \%$ and $0.8 \%$, respectively. In the simulations of the steady-state scenario, the diffusion coefficients for density and pressure are enhanced to $v_{\mathrm{n}}=\chi=10^{-5} v_{\mathrm{A}} R_{0}$ for $\sqrt{\psi_{\mathrm{p}}}>0.7$ to stabilize bulk pressure-driven MHD instabilities. Here, $\psi_{\mathrm{p}}$ is the normalized poloidal magnetic flux.

The time evolution of the MHD fluctuation energy for each toroidal mode number $n$ is shown in Fig. 3. In the early phase, the fluctuations with $n=13-17$ are dominant, and those with $n=3$ and 5 dominate at the later phase. The fluctuations with $n=13-17$ are the toroidal Alfvén eigenmodes (TAE modes), and those with $n=3$ and 5 are beta-induced Alfvén eigenmodes (BAE modes). The radial MHD velocity fluctuations multiplied by the minor radius coordinate $\left(r v_{\mathrm{r}}\right)$ at (a) $\mathrm{t}=1076 \omega_{\mathrm{A}}^{-1}$ and $(\mathrm{b}) \mathrm{t}=2111 \omega_{\mathrm{A}}^{-1}$ are shown in Fig. 4. The spatial profiles of the $n=15 \mathrm{TAE}$ 

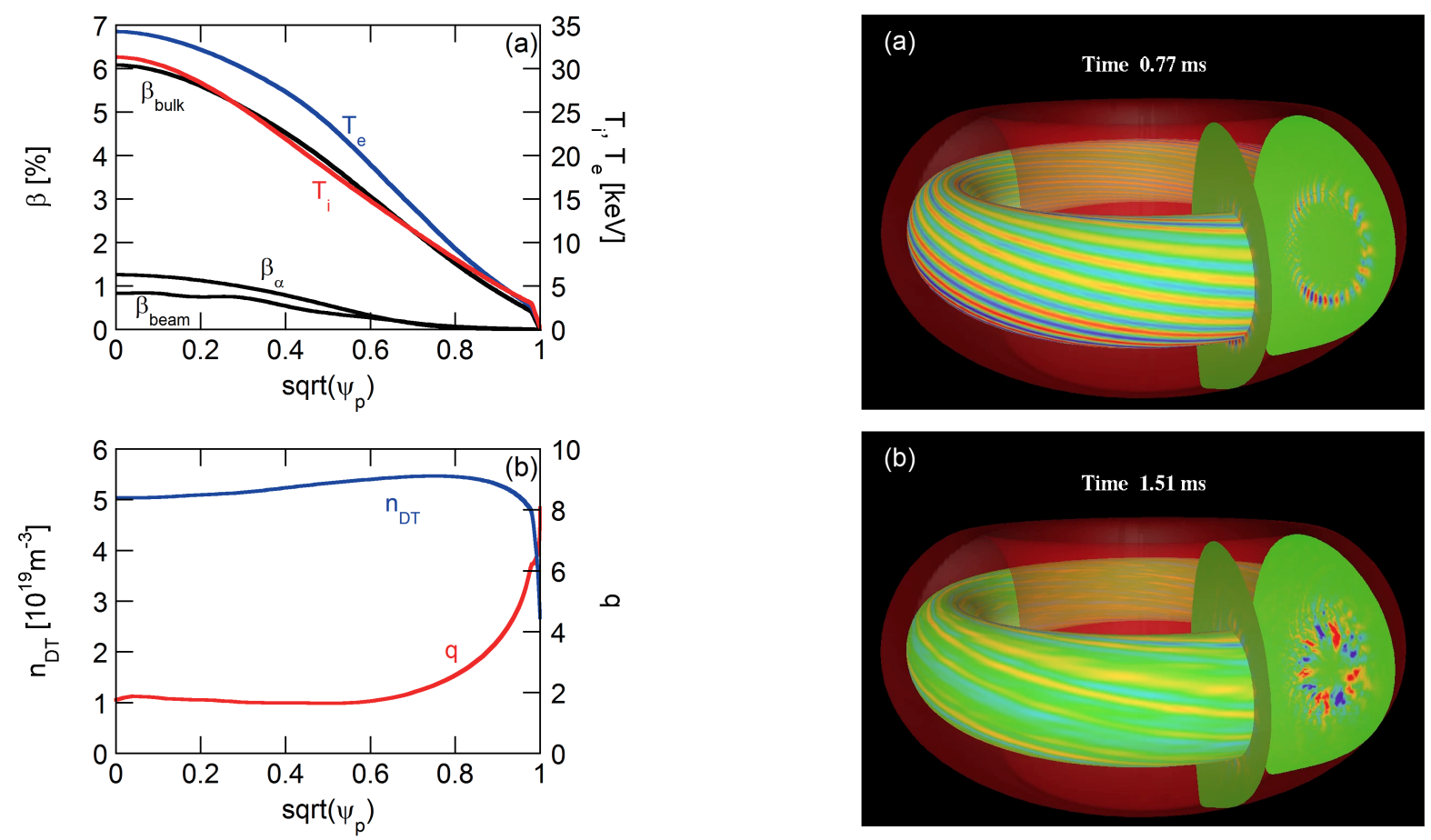

Fig. 2 Beta profiles of bulk, alpha, and beam ions, as well as the temperature profiles of ions and electrons (a) and the profiles of the deuterium-tritium density and safety factor for the ITER steady-state scenario with a $9 \mathrm{MA}$ plasma current. The horizontal axis is the square root of the normalized poloidal flux.

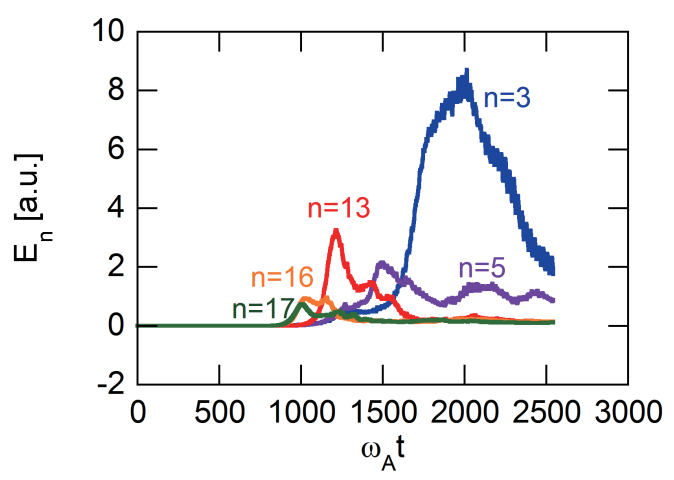

Fig. 3 Energy evolution of the MHD fluctuation for different dominant toroidal mode number $n$.

mode and the $n=3 \mathrm{BAE}$ mode are shown with the Alfvén continuous spectra in Figs. 5 and 6, respectively. The $n=3$ BAE mode forms peaks around the $q=5 / 3$ magnetic surface. The saturation levels of the radial velocity fluctuations for the $n=13$ TAE mode and the $n=3 \mathrm{BAE}$ mode are $v_{\mathrm{r}} / v_{\mathrm{A}} \sim 3 \times 10^{-3}$ and $v_{\mathrm{r}} / v_{\mathrm{A}} \sim 4 \times 10^{-3}$, respectively. Redistribution occurs for both energetic alpha particles and beam deuterium ions. The initial and final beta profiles are compared in Fig. 7. The reduction in beta value is $0.07 \%$ for the energetic alpha particles and the beam ions, which are $6 \%$ and $8 \%$ of the central beta values, respectively.

Fig. 4 Radial MHD velocity fluctuations multiplied by minor radius coordinate $\left(r v_{\mathrm{r}}\right)$ at (a) $t=1076 \omega_{\mathrm{A}}^{-1}$ and (b) $t=$ $2111 \omega_{\mathrm{A}}^{-1}$.
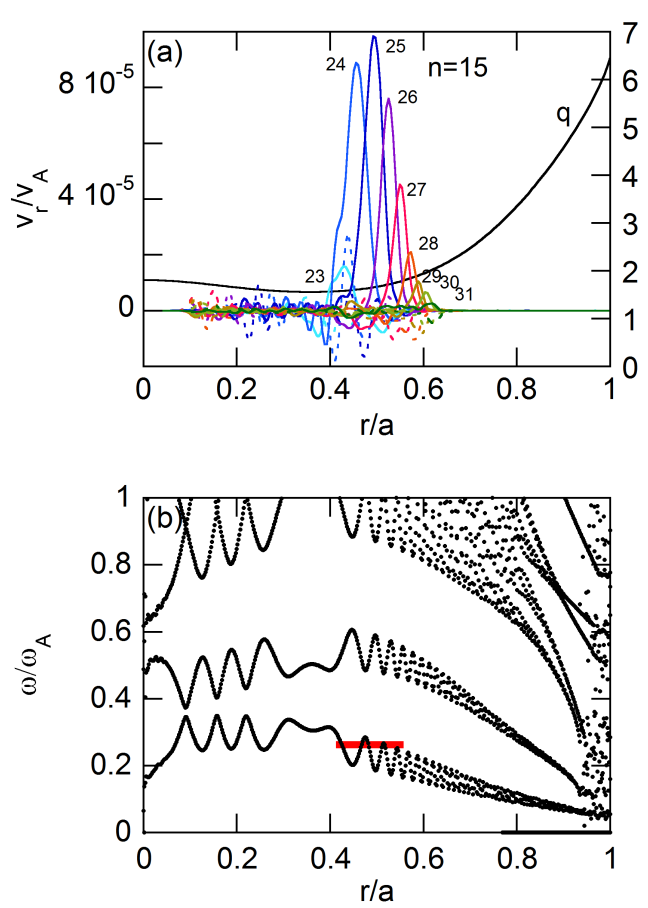

Fig. 5 Spatial profile of the $n=15$ TAE mode with the poloidal mode numbers shown in the figure (a) and the $n=15$ Alfvén continuous spectra with the frequency of the TAE mode shown with the red line (b).

\subsection{Convergence with particle number}

Another run, in which the number of computational particles increased to 34 million for each alpha particles 

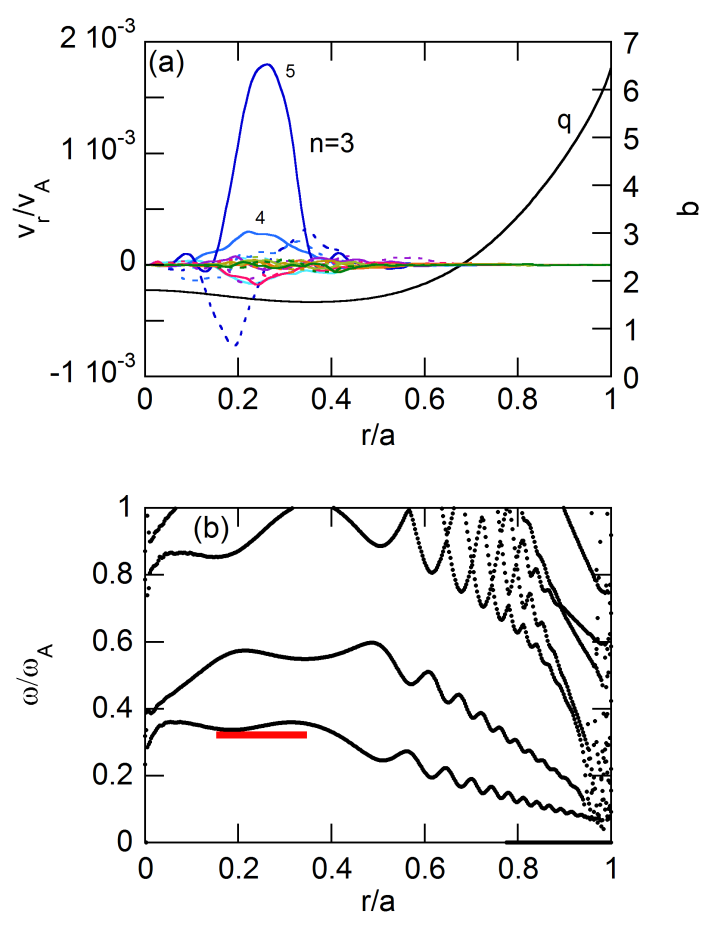

Fig. 6 Spatial profile of the $n=3$ BAE mode with the poloidal mode numbers shown in the figure (a) and the $n=3$ Alfvén continuous spectra with the frequency of the BAE mode shown by the red line (b).
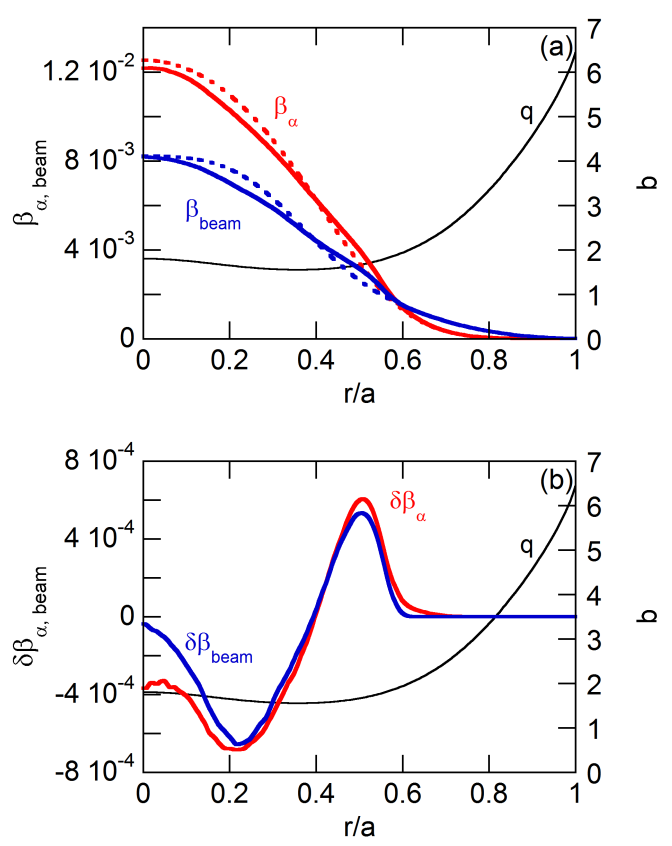

Fig. 7 Comparison of the initial and final beta profiles of the energetic alpha particles and beam deuterium ions (a) and the fluctuation profiles of the beta values (b).

and beam deuterium ions, was performed to check the numerical convergence. The number of particles is four times larger than the standard run discussed in the previous subsection. The results are shown in Fig. 8 in which we
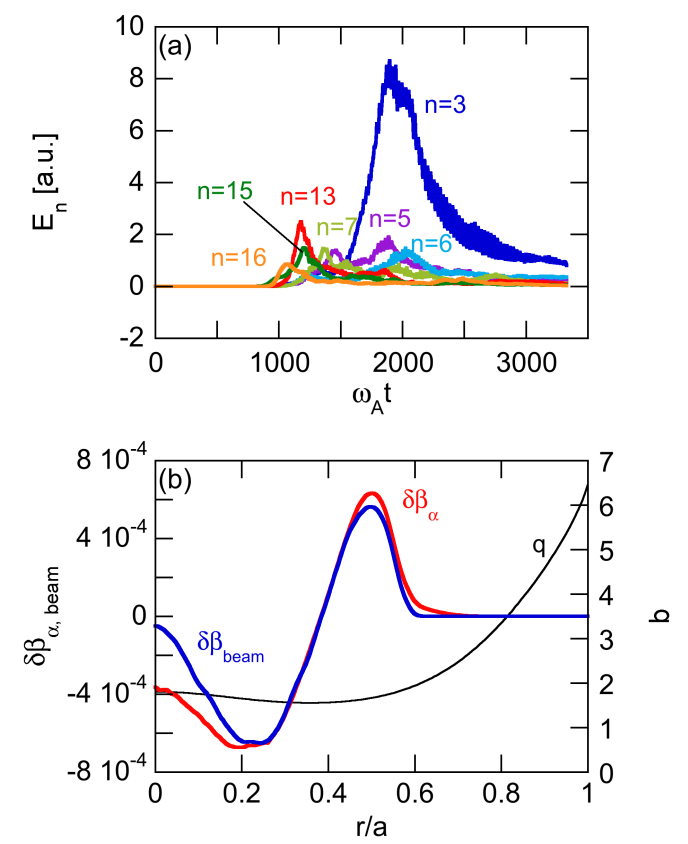

Fig. 8 Energy evolution of the MHD fluctuation for the dominant toroidal mode number (a) and the energetic particle beta fluctuation profiles (b) for the plasma with 9 MA current using 34 million particles for each alpha particle and beam ion.

show the energy evolution of the MHD fluctuations for the dominant toroidal mode number and the energetic particle beta fluctuation profiles at the end of the run. Comparing Figs. 8 (a) and 3, we observe in both figures that the largest fluctuation with $n=3$ has a maximum value of about eight in the unit of the figure at $t \sim 2000 \omega_{\mathrm{A}}^{-1}$. We observe in Fig. 8 (a) that the $n=13$ mode is the second largest fluctuation, and the fluctuations with $n=13-16$ increase before the growth of the $n=3$ mode. These are the same as that in Fig. 3. This indicates that the early growth of TAE modes for $n \sim 15$ is common between the two runs although the $n=17$ mode is weak and not shown in Fig. 8 (a).

For the beta fluctuation profiles of the energetic particles, we observe good agreement between Figs. 7 (b) and 8 (b). Thus, we conclude that we have good numerical convergence and the number of particles used is sufficient.

\subsection{Effects of multiple modes and plasma size}

The resonance overlap of the multiple AE modes enhances the energetic particle transport [18]. In the results presented in subsection 3.1, the TAE modes with $n=13$ 17 grow faster than the BAE modes with $n=3$ and 5 . The extent to which the TAE modes affect the growth of the BAE modes and the energetic particle transport is an interesting and important question.

To answer this question, we performed another run with the toroidal mode number of the MHD fluctuations restricted to $n \leq 8$. We imposed a numerical filter on the 

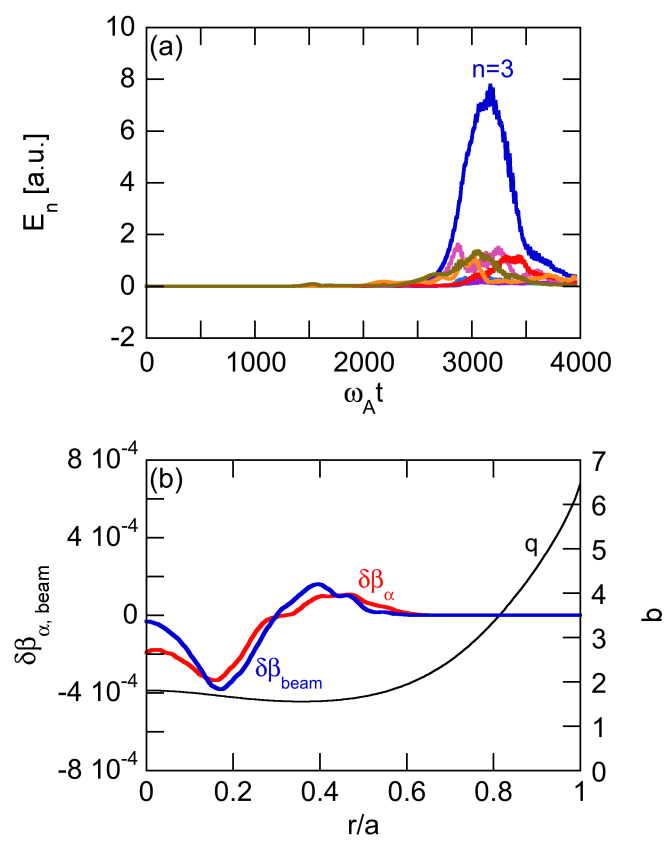

Fig. 9 Energy evolution of MHD fluctuation for each toroidal mode number; (a) and energetic particle beta fluctuation profiles; (b) in the simulation with toroidal mode numbers restricted to $n \leq 8$.

MHD fluctuations to remove the $n \geq 9$ modes. In this run, the TAE modes with $n=13-17$ are not destabilized and the comparison between these two runs clarifies the effect of the TAE modes. The energy evolution of each toroidal mode number is shown in Fig. 9(a). As observed in the standard run shown in Fig. 3, the BAE mode with $n=3$ grows faster than in the restricted run shown in Fig. 9 (a). The energetic particle beta fluctuations at the final state in the restricted run are shown in Fig. 9 (b). The beta value reduction is $0.03 \%$ and $0.04 \%$ in the restricted run for the energetic alpha particles and the beam deuterium ions, respectively, which corresponds to $2 \%$ and $5 \%$ of the central values. These values are lower than the $6 \%$ and $8 \%$ in the standard run. Thus, we conclude that the $n=13-17$ TAE modes enhance the growth in the BAE mode and the energetic particle redistribution.

One of the most important differences between ITER and present-day devices is the ratio of energetic particle orbit width to plasma size. We have performed another run by reducing the plasma size to $1 / 3$ of the ITER plasma, which is comparable to the present-day devices. The ratio of the energetic particle orbit width to the minor radius is enhanced by a factor of three. The energy evolution of the MHD fluctuations is shown in Fig. 10 (a) for each toroidal mode number. The most unstable mode number shifts to $n=3-9$ from $n=13-17$ in the ITER shown in Fig. 3 . The profiles of the energetic particle beta fluctuations are shown in Fig. 10 (b). The reduction in the energetic alpha particle beta is $0.04 \%$, which is less than the $0.07 \%$ reduction in the ITER plasma shown in Fig. 7 (b).
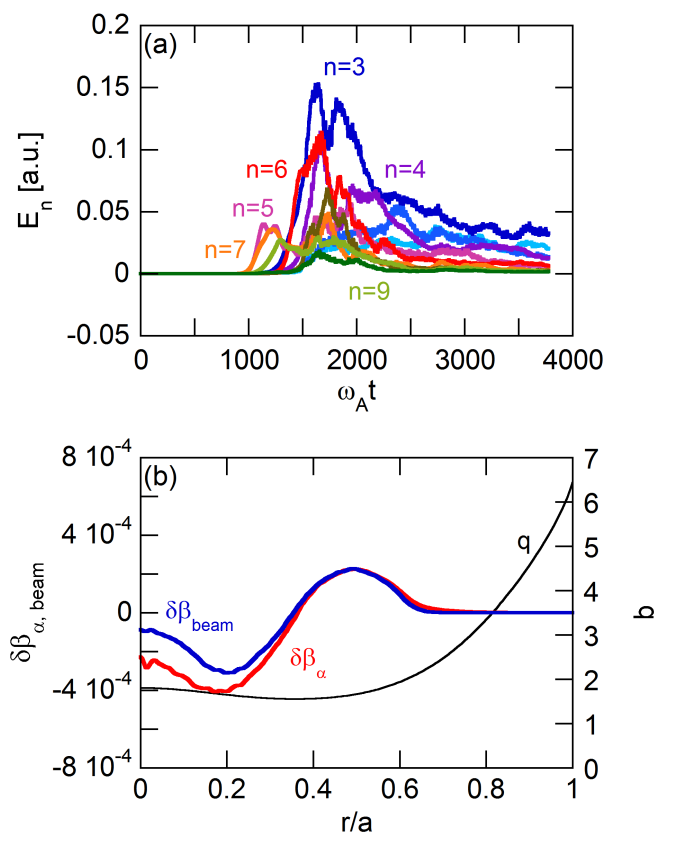

Fig. 10 Energy evolution of MHD fluctuation for each toroidal mode number; (a) and energetic particle beta fluctuation profiles; (b) in the 1/3-size plasma.

\subsection{MHD instability in monotonic shear plasma with 15 MA plasma current}

We have investigated monotonic magnetic shear plasma with $15 \mathrm{MA}$ plasma current [19]. The equilibrium data were obtained using the ASTRA and EFIT codes. The equilibrium plasma profiles are shown in Fig. 11. At the plasma center, the safety factor is slightly lower than one, and the safety factor is one $(q=1)$ at three plasma radii: $r / a=0.15,0.31$, and 0.38 . For such plasmas with triple $q=1$ surfaces, tearing-type instabilities with toroidal mode number higher than one $(n>1)$ were found [20]. We also observed that another type of strong MHD instability occurrs. The energy evolution of each toroidal mode number is shown in Fig. 12 (a). The instability has the toroidal mode number $n=3$. Figure 12 (b) shows the evolution of thermal, magnetic, fluid kinetic, energetic particle, and total energy. The thermal and magnetic energy decrease; this indicates that instability is driven by bulk pressure and plasma current. The energetic particle energy slowly increases suggesting that the energetic particles stabilize the instability. The total energy is kept almost zero, which indicates that the total energy is well conserved. The spatial profile of the instability is shown in Fig. 13 (a). The profile peaks around $r / a=0.3$ close to two of the $q=1$ surfaces. A similar instability with $n>1$ was also found for plasma profiles with relatively high magnetic shear at the $q=1$ surface [21]. The initial and final beta profiles of the energetic particles are compared in Fig. 13 (b). We observe a significant redistribution of the energetic alpha particle beta profile. The reduction in the alpha particle beta value is $0.3 \%$, which corresponds to a quarter of the 

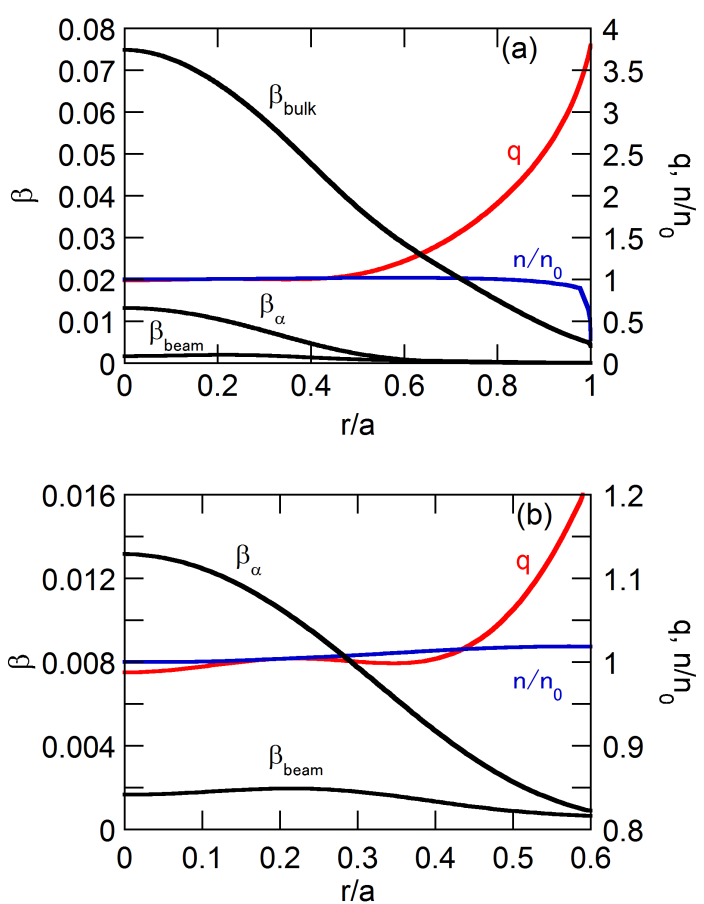

Fig. 11 Equilibrium profiles of an ITER scenario with $15 \mathrm{MA}$ plasma current; (a). Panel (b) is an enlargement for $0 \leq r / a \leq 0.6$ of panel (a).
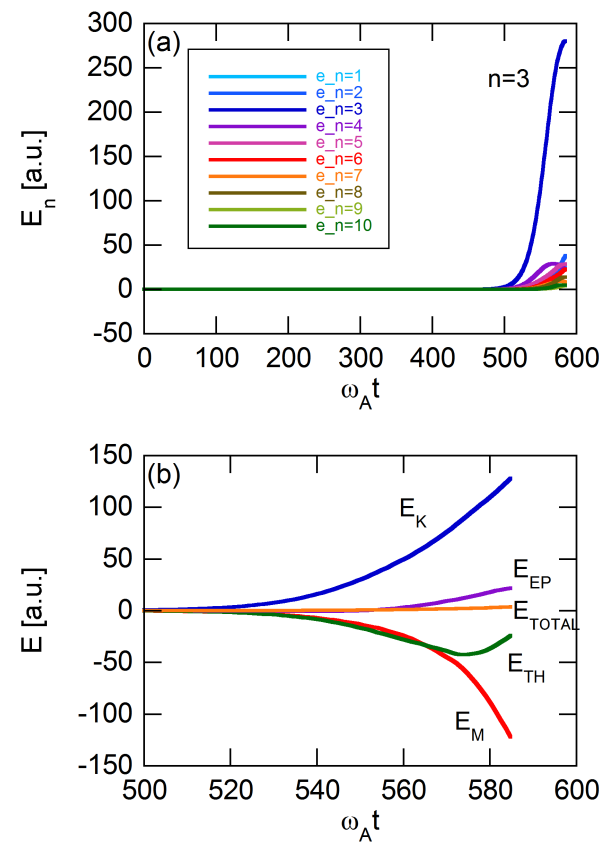

Fig. 12 Evolution of the MHD fluctuation energy for different toroidal mode numbers (a) and of the kinetic energy $\left(E_{\mathrm{K}}\right)$, magnetic energy $\left(E_{\mathrm{M}}\right)$, thermal energy $\left(E_{\mathrm{TH}}\right)$, energetic particle energy $\left(E_{\mathrm{EP}}\right)$, and total energy $\left(E_{\mathrm{TOTAL}}\right)(\mathrm{b})$.

central value. We performed another run with the safety factor profile uniformly raised by 0.1 to remove the $q=1$ surfaces. The evolution of the MHD fluctuations for each toroidal mode number is shown in Fig. 14 (a). The dom-
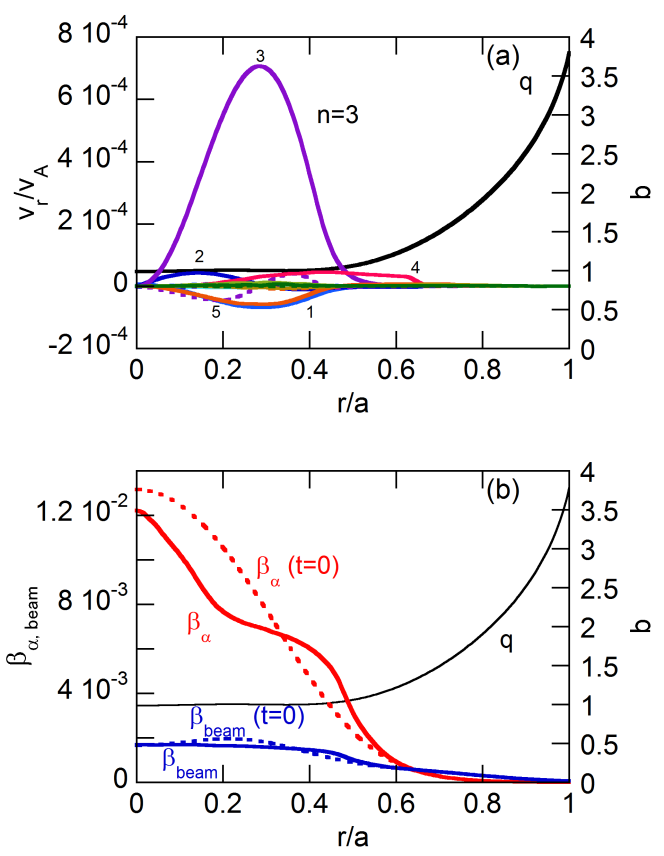

Fig. 13 Spatial profile of the $n=3$ MHD instability with the poloidal mode numbers shown in the figure (a) and comparison of the initial and final beta profiles of the energetic alpha particles and beam deuterium ions (b).

inant mode profile with $n=10$ is shown in Fig. 14 (b). In this case, we found only benign MHD instability, and neither significant MHD instability nor significant AE activity. The energetic particle beta profiles are compared in Fig. 14 (c) for the initial and final states. We see no substantial difference between the initial and final states. The energetic particle transport is negligible when there is no $q=1$ surface.

\section{Summary}

We have investigated the evolution of $\mathrm{AE}$ and the associated energetic particle transport, and the ideal MHD instabilities for the ITER operation scenarios using the MEGA code. The particle simulation method with the finite Larmor radius effects was applied to the energetic alpha and beam deuterium particles. For steady-state plasma with 9 MA plasma current, the beta-induced Alfvén eigenmodes (BAE modes) with low toroidal mode number $(n=$ $3 \& 5$ ) are found to be dominant in the nonlinear phase although many toroidal Alfvén eigenmodes (TAE modes) with $n=15$ are most unstable in the linear phase. The redistribution of energetic particles with $\delta \beta_{\alpha} \sim \delta \beta_{\text {beam }} \sim$ $0.07 \%$ occurs in the nonlinear phase. When the toroidal mode number of the fluctuations is restricted to $n \leq 8$, the redistribution substantially decreases. This suggests that the resonance overlap between the TAE modes with $n \sim 15$ and the low- $n$ BAE modes enhances the energetic particle transport.

For ITER plasma with $15 \mathrm{MA}$ plasma current, the 

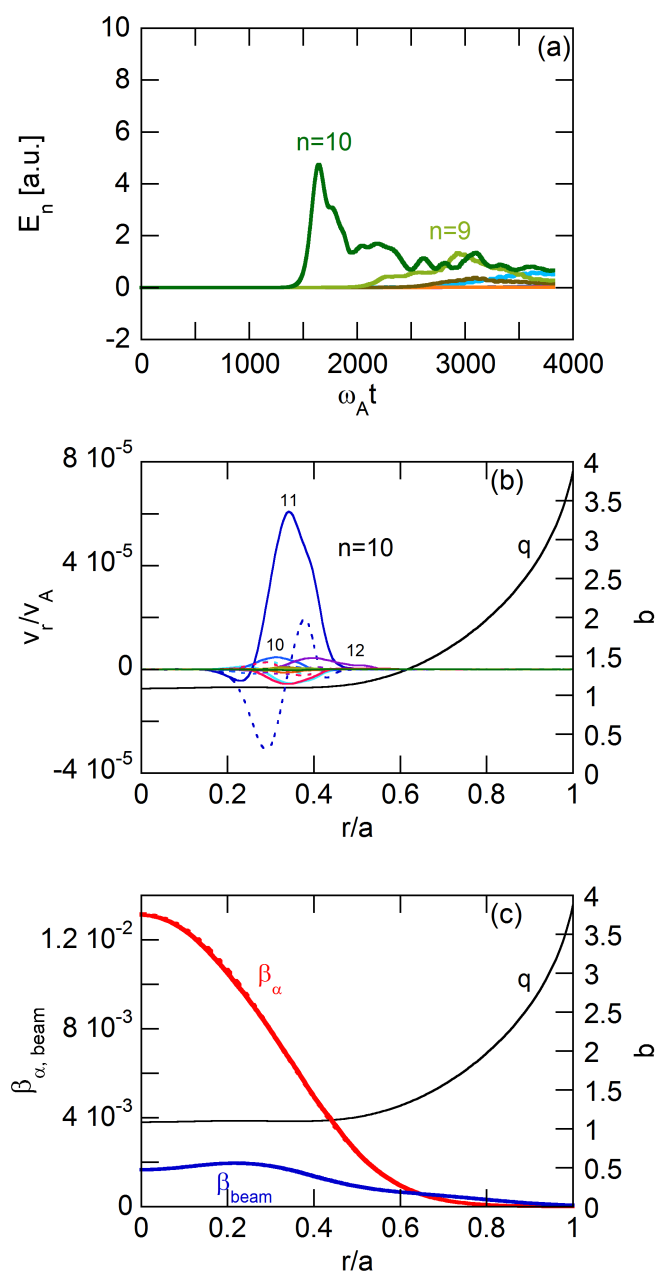

Fig. 14 Energy evolution of the MHD fluctuations for different toroidal mode numbers $n$ (a), spatial profile of the $n=10$ MHD instability (b), and comparison of the initial and final beta profiles of the energetic alpha particles and beam deuterium ions (c) in an ITER-like equilibrium with the $q$ value uniformly raised by 0.1 .

MHD instability with $n=3$ results in significant redistribution of the alpha particles with $\delta \beta_{\alpha} \sim 0.3 \%$. When the safety factor profile is uniformly raised by 0.1 to remove the $q=1$ magnetic surfaces, only benign MHD instability occurs and the energetic particle transport is negligible.

The simulations presented in this study do not include the kinetic damping of the $\mathrm{AE}$ mode, such as radiative damping [22] and thermal ion Landau damping. The simulations overestimate the growth rate of the AE modes and the associated energetic particle transport. We regard the results of this study as an upper limit of the redistributions of the energetic particles. The redistributions in the simulations are benign in the steady-state $9 \mathrm{MA}$ scenario and negligible in the $15 \mathrm{MA}$ scenario if the $q=1$ magnetic surfaces are avoided. The results obtained are encouraging for future ITER experiments.

\section{Acknowledgments}

Numerical computations were performed using the Helios of the International Fusion Energy Center, the Plasma Simulator of National Institute for Fusion Science, and the K Computer of the RIKEN Advanced Institute for Computational Science (Project ID: hp120212). This study was supported by JSPS and leading research organizations, namely NSERC, ANR, DFG, RFBR, RCUK, and NSF as Partner Organizations under the G8 Research Councils Initiative for Multilateral Research Funding. This study was also supported by the JSPS-NRF-NSFC A3 Foresight Program in the field of Plasma Physics (NSFC: No.11261140328).

[1] N.N. Gorelenkov, H.L. Berk and R.V. Budny, Nucl. Fusion 45, 226 (2005).

[2] G. Vlad, S. Briguglio, G. Fogaccia, F. Zonca and M. Schneider, Nucl. Fusion 46, 1 (2006).

[3] Y. Todo, J. Plasma Phys. 72, 817 (2006).

[4] M.A. Van Zeeland et al., Nucl. Fusion 52, 094023 (2012).

[5] Y. Todo and T. Sato, Phys. Plasmas 5, 1321 (1998).

[6] Y. Todo, K. Shinohara, M. Takechi and M. Ishikawa, Phys. Plasmas 12, 012503 (2005).

[7] Y. Todo, Phys. Plasmas 13, 082503 (2006).

[8] A. Könies et al., Benchmark of gyrokinetic, kinetic MHD and gyrofluid codes for the linear calculation of fast particle driven TAE dynamics, in Proc. 24th IAEA Fusion Energy Conference (Oct. 8-13, 2012, San Diego) ITR/P1-34.

[9] G. Pereverzev, P.N. Yushmanov, ASTRA Automated System for Transport Analysis in a Tokamak, IPP-Report, IPP $5 / 98(2002)$

[10] L.L. Lao et al., Nucl. Fusion 25, 1421 (1985).

[11] W. Park et al., Phys. Fluids B 4, 2033 (1992).

[12] D.A. Spong, B.A. Carreras and C.L. Hedrick, Phys. Fluids B 4, 3316 (1992).

[13] Y. Todo, T. Sato, K. Watanabe, T.H. Watanabe and R. Horiuchi, Phys. Plasmas 2, 2711 (1995).

[14] S. Briguglio, G. Vlad, F. Zonca and C. Kar, Phys. Plasmas 2, 3711 (1995).

[15] X. Wang, F. Zonca and L. Chen, Plasma Phys. Control. Fusion 52, 115005 (2010).

[16] R.G. Littlejohn, J. Plasma Phys. 29, 111 (1983).

[17] IDM DATA folder: Plant Breakdown Structure/ TBD. Plasma/10.1.1 Plasma Confinement/ EnergeticParticles/ ITER reference data for EP modeling/Equilibrium/ Update2011/9MA plasma equilibrium

[18] H.L. Berk, B.N. Breizman and M.S. Pekker, Nucl. Fusion 35, 1713 (1995).

[19] IDM DATA folder: Plant Breakdown Structure/ TBD. Plasma/10.1.1 Plasma Confinement/ EnergeticParticles/ ITER reference data for EP modeling/Equilibrium/ Update2011/15MA plasma equilibrium

[20] A. Bierwage et al., Phys. Rev. Lett. 94, 065001 (2005).

[21] Z.-X. Wang, L. Wei and X. Wang, Phys. Plasmas 19, 062108 (2012)

[22] R.R. Mett and S.M. Mahajan, Phys. Fluids B 4, 2885 (1992). 